Pacific Journal of Mathematics

SIMPLE POINTS IN PSEUDOLINE ARRANGEMENTS 


\section{SIMPLE POINTS IN PSEUDOLINE ARRANGEMENTS}

\section{M. Kelly AND R. Rottenberg}

A finite collection of simple closed curves in the real projective plane each two of which have exactly one point in common at which point they "cross" is called a pseudoline arrangement. Such arrangements seem to have been first systematically studied by Levi. Recently B. Grünbaum has called attention to the desirability for a better understanding of the differences as well as the similarities in the behavior of arrangements of lines and the arrangements of pseudolines.

Among other things, Grünbaum asks if every pseudoline arrangement, not all curves of which intersect in a single point, must have a simple vertex (a point on exactly two of the curves of the arrangement) as is the case in line arrangements. In fact Kelly and Moser have shown that, in general, an $n$-line arrangement in an ordered projective plane has at least $3 / 7 n$ simple vertices. It is shown here that their reasoning can be nearly dualized to prove the analogous result for pseudoline arrangements.

However we make heavy use of a lemma of Levi proved in [4]. Examples show that it is possible to realize pseudoline arrangements which are not combinatorially equivalent to any line arrangement.

2. Preliminaries, definitions, notation.

Definition 2.1. A finite collection, $F$, of simple closed curves in the real projective plane, each two of which have exactly one point in common, at which point they cross, is called a pseudoline arrangement. Each curve is called a pseudoline or, where there is no danger of confusion merely a line. The set of points of intersection of the lines of $F$ is denoted $I(F)$ and each point of $I(F)$ is a vertex of $F$. If exactly two lines of $F$ pass through a vertex it is simple or ordinary.

LEMMA OF LEVI. If $F$ is a pseudoline arrangement in the real projective plane $\mathscr{P}$ and $A$ and $B$ any two points of $\mathscr{P}$, then there exists a simple closed curve, $g$, containing $A$ and $B$ such that $F \cup\{g\}$ is a pseudoline arrangement.

By successive applications of this lemma we obtain a pseudoline arrangement containing $F$ such that each two points of $I(F)$ are on exactly one line of the arrangement. Each line of $F$ is intersected by the lines of this extended arrangement in a finite number of points 
partitioning the line into a finite number of open arcs. Let $S$ be a set of points contained in the union of such arcs with exactly one point on each arc.

We now further extend the arrangement so that each point of $S$ is joined by a pseudoline to each point of $I(F)$. This enlarged arrangement is denoted $F^{*}$. We are now able to confine our attention to this arrangement $F^{*}$.

We need some of the analogues of the order and separation properties of projective lines in the pseudolines of our arrangement $F$.

If $A_{1}, A_{2}, A_{3}, A_{4}$ are four points on a simple closed curve $\Gamma$ and if $A_{1}$ and $A_{2}$ are in different components of $\Gamma-\left\{A_{3}, A_{4}\right\}$ we say that $A_{1}$ and $A_{2}$ separate $A_{3}$ and $A_{4}$ and write $A_{1}, A_{2} \| A_{3}, A_{4}$. The usual separation axioms for separation on the real projective line are well known to be satisfied e.g. $A_{1}, A_{2}\left\|A_{3}, A_{4} \Rightarrow A_{3}, A_{4}\right\| A_{1}, A_{2}$.

If $g_{1}, g_{2}, g_{3}, g_{4}$ are four pseudolines through a point $P$ in an arrangement $F$ and if $g_{1}-\{P\}$ and $g_{2}-\{P\}$ are in different components of $\mathscr{P}-\left(g_{3} \cup g_{4}\right)$ then $g_{1}$ and $g_{2}$ are said to separate $g_{3}$ and $g_{4}$; This separation is similarly symbolized $g_{1}, g_{2} \| g_{3}, g_{4}$.

If $l$ is a line of $F$ not through $P$ and $g_{i} \cap l=A_{i}$ it follows by conventional arguments that $g_{1}, g_{2} \| g_{3}, g_{4}$ iff $A_{1}, A_{2} \| A_{3}, A_{4}$. This implies that if $l^{\prime}$ is a second line of $F$ not through $p$ with $l^{\prime} \cap g_{i}=A_{i}$ then $A_{1}, A_{2} \| A_{3}, A_{4}$ iff $A_{1}^{\prime}, A_{2}^{\prime} \| A_{3}^{\prime}, A_{4}^{\prime}$ i.e., separation on pseudolines is a "perspective invariant" in $F$.

Two points of $I(F) \cup S$ are joined by at most one line of $F^{*}$. If $P$ and $Q$ are two points so joined we denote this unique line of $F^{*}$ by $P Q$. If $A$ is a point of $S$ and $K$ a subset of $I(F)$ then the pencil $\{A X \mid X \in K\}$ is denoted $[A: K]$. A set of lines all but one of which go through a point is a near pencil.

Definition 2.2. A point $P \in I(F)$ is a neighboring point (or simply a neighbor) of $l \in F$ iff there exists a point $A \in S$ on $l$ such that $A P$ and $l$ are distinct and separate no two lines in the pencil $[A: I(F)] . \quad P$ is said to be a neighbor of $l$ relative to $A$ in $F^{*}$.

\section{The principal theorem.}

THEOREM 3.1. If $F$ is not a near pencil then each line of $F$ has at least three neighbors in $I(F)$.

Proof. Let $l$ be any line of $F$ and $A \in S \cap l$. The pencil $[A: I(F)]$ contains a pair of lines $A P$ and $A Q$ separating $l$ from any other line in the pencil. $P$ and $Q$ are, by definition, neighbors of $l$ relative to $A$.

Since $F$ is not a near pencil, $P$ and $Q$ are distinct and we denote the line $P Q$ by $t$. Furthermore, for the same reason, there exists a 
point $R \in I(F)$ not on $t \cup l$. Let $R P \cap l=R^{\prime}, t \cap l=Q^{\prime}$ and $B$ a point of $S$ on the arc $R^{\prime} Q^{\prime}$ which does not contain $A$.

Let $A R \cap t=V$ and $R B \cap t=M$. By the definition of neighbor it follows that $V$ and $Q$ lie together on the same segment of $t$ with end point $P$ and $Q^{\prime}$. On the other hand $A, B\left\|Q^{\prime} R^{\prime} \Rightarrow V, M\right\| Q^{\prime}, P$ which means that $V$ and $M$ are on complementary segments of $t$ with end points $P$ and $Q^{\prime}$. Thus $M$ and $Q$ are on complementary segments with end points $P$ and $Q^{\prime}$, i.e., $M, Q \| P, Q^{\prime}$ or $B P, B Q \| B P, l$. Hence $P$ is not a neighbor of $l$ relative to $B$.

But, as above, there are two neighbors of $l$ relative to $B$ and it follows that $l$ has at least three neighbors.

Theorem 3.2. If $P \in I(F)$ is a neighbor of three lines $l, m, n$ of $F$ then any line of $F$ through $P$ goes through one of the points $l \cap m$, $l \cap n, m \cap n$.

Proof. Suppose $t$ a line of $F$ through $P$, with $t \cap l=A t \cap m=B$, $t \cap n=C$. Suppose that $A, B, C$ are distinct and that $P$ and $A$ separate $B$ and $C$. Since $P$ is a neighbor of $l$, there is a point $X \in S$ on $l$ such that $X P$ and $l$ separate no pair of lines of the pencil $[X: I(F)]$. But $X P$ and $l$ separate $X B$ and $X C$ for any $X \in S$ on $l$. Hence $t$ must pass through one of the points $l \cap m, l \cap n, m \cap n$.

CoRollary 3.2.1. Three or more lines of $F$ meeting at one point cannot have a common neighbor.

THEOREM 3.3. If $P \in I(F)$ is a neighbor of a line $l$ of $F$, then at most two of the points of intersection of $l$ with lines of $F$ through $P$ are not simple.

Proof. The theorem is obvious if $P$ is simple. Suppose, then, that $m_{1}, m_{2}, \cdots, m_{k}, k>2$, are lines of $F$ through $P$ and that $l \cap m_{i}=$ $M_{i}$ for each $i$. If $P$ is a neighbor of $l$ relative to the point $X$ we may assume that $M_{1}$ and $M_{2}$ separate $X$ from each of the other $M_{i}$ $i=3,4, \cdots, k$. Assume that $M_{j}, j>2$, is not simple and that $t$ is a third line (in addition to $m_{j}$ and $l$ ) through $M_{j}$. If $X^{\prime}=t \cap X P$ $M_{1}^{\prime}=m_{1} \cap t$ and $M_{2}^{\prime}=m_{2} \cap t$ then $M_{1}^{\prime}, M_{2}^{\prime} \| X^{\prime}, M_{j}$ which implies that $X M_{1}^{\prime}$ and $X M_{2}^{\prime}$ separate $X P$ and $l$. Thus $P$ is not a neighbor of $l$ relative to $X$. This contradiction means that each $M_{j}, j>2$, is simple and proves the theorem.

Corollary 3.3.1. If $P$ is a neighbor of four lines of $F$, then $P$ is a vertex of the diagonal triangle of the complete quadrilateral defined by the four lines. Furthermore $P$ is simple, the two lines of $F$ through $P$ being the diagonal lines of the quadrilateral. 
Proof. If $P$ is a neighbor of the four lines $l_{1}, l_{2}, l_{3}, l_{4}$ it follows from Corollary 3.2.1 that no three of these lines can be concurrent and the four lines form a complete quadrilateral, $K$.

By Theorem 3.2 any line of $F$ through $P$ must go through a pair of opposite vertices of $K$. Thus since there are certainly two (distinct) such lines, $P$ is a vertex of the diagonal triangle of $K$.

If there were a third line of $F$ through $P$ it would have to be the third side of the diagonal triangle. But since the vertices of this triangle are certainly not simple we would have a contradiction of Theorem 3.3 and it follows that $P$ must be simple.

Corollary 3.3.2. A point $P$ of $I(F)$ is a neighbor of at most four lines of $F$.

Definition 3.1. The number of simple points of $I(F)$ on a line $l$ of $F$ is the order of $l$. The number of neighbors of $l$ which are simple is the rank of $l$. The order plus the rank is the index.

THEOREM 3.4. The index of each line of $F$ which is not of order 2 is at least 3 .

Proof. First observe that the theorem is true for a near pencil and dismiss this case from further consideration.

Case 1. The order of $l$ is zero. By Theorem 3.3 every neighbor of $l$ must be simple and by Theorem 3.1 the rank, hence the index, of $l$ is at least 3 .

Case 2. The order of $l$ is 1 .

Suppose $P_{1}$ and $P_{2}$ two nonsimple neighbors of $l, M$ the line simple point on $l$ and $m$ the second line of $F$ through $M$. It follows from Theorem 3.3 that there are exactly three lines of $F$ through each of the points $P_{i}$ one of which must go through $M$ and the other two of which meet $l$ in the distinct points $A_{i}$ and $B_{i}$.

Since $M$ is simple $P_{1} M=P_{2} M=m$. Now if $P_{3} \neq P_{1}, P_{2}$ or $M$ is a point on $m$ then for some permutation $(i, j, k)$ of $(1,2,3), P_{i}, M \| P_{j}, P_{k}$ from which if follows that $P_{3}$ cannot be a neighbor of $l$. We can now conclude that there are at most two nonsimple neighbors of $l$. It is easy to see that there exists a point $Y \in S$ on $l$ such that, subject to proper labelling of $A_{i}$ and $B_{i}, Y, A_{i} \| M, B_{i}$. Since $A_{i}$ is nonsimple there is a line $n \in F$ distinct from $P_{i} A_{i}$ and $l$. Let $n \cap P_{i} Y=$ $X, n \cap m=Z, n \cap P_{i} B_{i}=W$.

$$
Y, A_{i}\left\|M, B_{i} \Rightarrow X, A_{i}\right\| Z, W \Rightarrow Y P_{i}, l \| Y Z, Y W \text {. }
$$




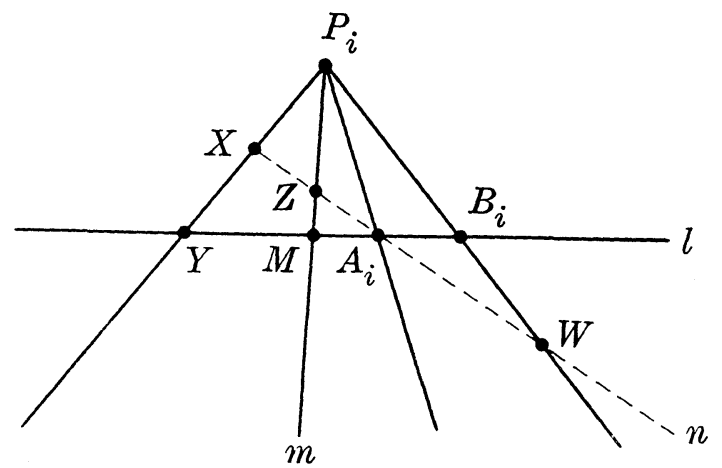

FIGURE 1

Thus $P_{i}$ is not a neighbor of $l$ relative to point $Y$.

But as in Theorem 3.1 there are two distinct neighbors of $l$ relative to $Y$, both of which are simple. Thus the index of $l$ is at least 3.

Case 3. The order of $l$ is at least 3. The index of $l$ is, by definition, at least 3 .

THEOREM 3.5. If $\lambda_{i}$ is the index of line $l_{i} \in F$, then $\lambda \geqq 1 / 6 \sum_{1}^{n} \lambda_{i}$ where $\lambda$ is the number of simple points of $I(F)$.

Proof. We count the number of simple points by observing the index for each of the $n$ lines of $F$. In this count a particular simple point may be counted as many as six times, four as a neighbor and two because it lies on two lines of $F$.

THEOREM 3.6.

$$
\lambda \geqq \frac{3}{7} n
$$

Proof. Suppose that there are $k$ lines of order 2. Clearly $\lambda \geqq k$. By Theorem 3.4 and 3.5,

$$
\lambda \geqq \frac{3(n-k)+2 k}{6}=\frac{3 n-k}{6} .
$$

Elimination of $k$ gives the desired result.

REMARK. That the inequality is sharp is shown by the seven lines forming a complete quadrilateral and its diagonal triangle. The three vertices of the diagonal triangle are the only simple points of the configuration. 


\section{REFERENCES}

1, B. Grünbaum, The importance of being straight, Proc. 12th Biennial Int. Sem. of the Can. Math. Congress, Vancouver, 1969.

2. — Polytopes, Graphs and Complexes, Bull. Amer. Math. Soc., 76 No. 6 (1970), 1131-1201.

3. L. M. Kelly and W. O. J. Moser, On the number of ordinary lines determined by $n$ points; Canad. J. Math., 10 (1958), 210-219.

4. F. Levi, Die Teilung der projective Ebene durch Gerade oder Pseudogerade, Ber. Math.-Phys, Kl. Sachs. Akad. Wiss. Leipzig, 78 (1926), 256-267.

Received February 16, 1971, and in revised form June 16, 1971.

Michigan State UNIVERSity 


\section{PACIFIC JOURNAL OF MATHEMATICS}

\section{EDITORS}

\section{H. SAMELSON}

Stanford University

Stanford, California 94305

\section{R. HOBBY}

University of Washington Seattle, Washington 98105

\section{J. DugundJI}

Department of Mathematics University of Southern California Los Angeles, California 90007

RICHARD ARENS

University of California Los Angeles, California 90024

\section{ASSOCIATE EDITORS}
E. F. BECKENBACH
B. H. Neumann
F. WoLF
K. YoSHIDA

\section{SUPPORTING INSTITUTIONS}

UNIVERSITY OF BRITISH COLUMBIA CALIFORNIA INSTITUTE OF TECHNOLOGY

UNIVERSITY OF CALIFORNIA

MONTANA STATE UNIVERSITY

UNIVERSITY OF NEVADA

NEW MEXICO STATE UNIVERSITY

OREGON STATE UNIVERSITY

UNIVERSITY OF OREGON

OSAKA UNIVERSITY

\author{
UNIVERSITY OF SOUTHERN CALIFORNIA \\ STANFORD UNIVERSITY \\ UNIVERSITY OF TOKYO \\ UNIVERSITY OF UTAH \\ WASHINGTON STATE UNIVERSITY \\ UNIVERSITY OF WASHINGTON \\ * * * * \\ AMERICAN MATHEMATICAL SOCIETY \\ NAVAL WEAPONS CENTER
}

The Supporting Institutions listed above contribute to the cost of publication of this Journal, but they are not owners or publishers and have no responsibility for its content or policies.

Mathematical papers intended for publication in the Pacific Journal of Mathematics should be in typed form or offset-reproduced, (not dittoed), double spaced with large margins. Underline Greek letters in red, German in green, and script in blue. The first paragraph or two must be capable of being used separately as a synopsis of the entire paper. The editorial "we" must not be used in the synopsis, and items of the bibliography should not be cited there unless absolutely necessary, in which case they must be identified by author and Journal, rather than by item number. Manuscripts, in duplicate if possible, may be sent to any one of the four editors. Please classify according to the scheme of Math. Rev. Index. to Vol. 39. All other communications to the editors should be addressed to the managing editor, Richard Arens, University of California, Los Angeles, California, 90024.

50 reprints are provided free for each article; additional copies may be obtained at cost in multiples of 50 .

The Pacific Journal of Mathematics is published monthly. Effective with Volume 16 the price per volume (3 numbers) is $\$ 8.00$; single issues, $\$ 3.00$. Special price for current issues to individual faculty members of supporting institutions and to individual members of the American Mathematical Society: $\$ 4.00$ per volume; single issues $\$ 1.50$. Back numbers are available.

Subscriptions, orders for back numbers, and changes of address should be sent to Pacific Journal of Mathematics, 103 Highland Boulevard, Berkeley, California, 94708.

PUBLISHED BY PACIFIC JOURNAL OF MATHEMATICS, A NON-PROFIT CORPORATION

Printed at Kokusai Bunken Insatsusha (International Academic Printing Co., Ltd.), 270, 3-chome Totsuka-cho, Shinjuku-ku, Tokyo 160, Japan. 


\section{Pacific Journal of Mathematics}

\section{Vol. 40, No. $3 \quad$ November, 1972}

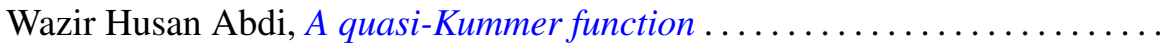

Vasily Cateforis, Minimal injective cogenerators for the class of modules of

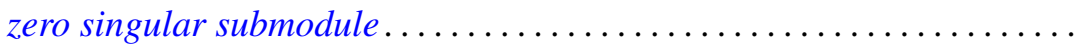

W. Wistar (William) Comfort and Anthony Wood Hager, Cardinality of

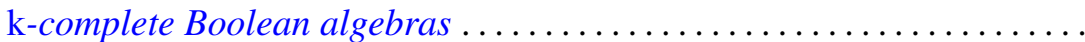

Richard Brian Darst and Gene Allen DeBoth, Norm convergence of martingales of Radon-Nikodym derivatives given a $\sigma$-lattice ..........

M. Edelstein and Anthony Charles Thompson, Some results on nearest points and support properties of convex sets in $c_{0} \ldots \ldots \ldots \ldots \ldots$

Richard Goodrick, Two bridge knots are alternating knots .

Jean-Pierre Gossez and Enrique José Lami Dozo, Some geometric properties related to the fixed point theory for nonexpansive mappings ..........

Dang Xuan Hong, Covering relations among lattice varieties .............

Carl Groos Jockusch, Jr. and Robert Irving Soare, Degrees of members of $\Pi_{1}^{0}$

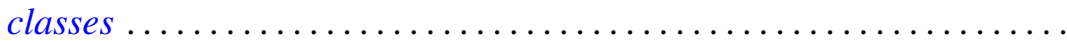

565

575

605

Leroy Milton Kelly and R. Rottenberg, Simple points in pseudoline

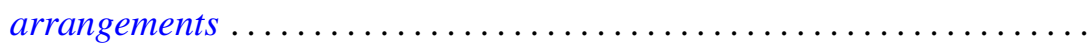

Joe Eckley Kirk, Jr., The uniformizing function for a class of Riemann surfaces....

Glenn Richard Luecke, Operators satisfying condition $\left(G_{1}\right)$ locally ... 629

T. S. Motzkin, On L $(S)$-tuples and l-pairs of matrices ... . .

Charles Estep Murley, The classification of certain classes of torsion free

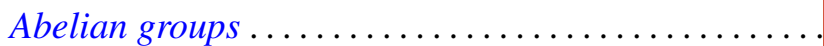

Louis D. Nel, Lattices of lower semi-continuous functions and associated topological spaces.

David Emroy Penney, II, Establishing isomorphism between tame prime

knots in $E^{3}$. . .

Daniel Rider, Functions which operate on $\mathscr{F} L_{p}(T), 1<p<2$

Thomas Stephen Shores, Injective modules over duo rings ...

Stephen Simons, A convergence theorem with boundary. .

703

Stephen Simons, Maximinimax, minimax, and antiminimax theorems and a

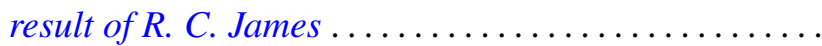

Stephen Simons, On Ptak's combinatorial lemma ........

Stuart A. Steinberg, Finitely-valued $f$-modules............

Pui-kei Wong, Integral inequalities of Wirtinger-type and fourth-order

elliptic differential inequalities .

Yen-Yi Wu, Completions of Boolean algebras with partially additive

operators ..................................

Phillip Lee Zenor, On spaces with regular $G_{\delta}$-diagonals . . . 